\title{
Circuit
}

Musiques contemporaines

\section{L’aventure du disque de musique québécoise}

Bilan d'une décennie et perspectives d'avenir

Recording Québécois Concert Music

An Adventure Report on a Decade of Activities and Future

\section{Prospects}

\section{Françoise Davoine}

Volume 1, numéro 2, 1990

Montréal musiques actuelles

URI : https://id.erudit.org/iderudit/902019ar

DOI : https://doi.org/10.7202/902019ar

Aller au sommaire du numéro

Éditeur(s)

Les Presses de l'Université de Montréal

ISSN

1183-1693 (imprimé)

1488-9692 (numérique)

Découvrir la revue

Citer cet article

Davoine, F. (1990). L'aventure du disque de musique québécoise : bilan d'une décennie et perspectives d'avenir. Circuit, 1(2), 81-92.

https://doi.org/10.7202/902019ar
Résumé de l'article

L'article présente un bilan statistique des parutions discographiques de compositeurs québécois selon chacune des étiquettes et analyse le contenu de la production des quatre plus importantes d'entre elles (McGill University Records, Centredisques, SNE et Société et Entreprises Radio-Canada). L'auteure évalue l'impact de la disparition de Radio-Canada International et dresse un bilan passablement pessimiste des perspectives d'avenir. 


\section{L'aventure du disque de musique québécoise : bilan d'une décennie et perspectives d'avenir Françoise Davoine}

Le marché québécois du disque de musique dite sérieuse a toujours eu des proportions limitées, on s'en doute. A fortiori celui du disque de musique $d u x x^{e}$ siècle. Et lorsqu'il s'agit de cerner la production de disques de musique de compositeurs québécois contemporains ${ }^{(1)}$, on peut d'autant moins s'attendre à un résultat d'envergure.

Pourtant, établir un catalogue exhaustif de cette production de 1980 à 1990 reste en soi une aventure. Certes, toute parution sur disque doit être légalement déposée à la Bibliothèque nationale du Canada, et ce depuis 1969. Mais il n'est actuellement pas possible d'extraire de cet ensemble la liste qui nous intéresse ${ }^{(2)}$. Le Centre de musique canadienne (CMC), quant à lui, recense tout ce qui concerne ses membres, bien sûr, mais les catalogues de disques publiés donnent la liste des enregistrements disponibles au $C M C$, et pas nécessairement de tout ce qui a été réalisé sur disque. Le corpus ainsi délimité ne rend donc pas justice à l'ensemble des compositeurs québécois. Reste la discothèque de Radio-Canada à Montréal qui semble être, pour l'instant encore, l'avenue la plus sûre, puisque, en plus des parutions commerciales, elle compte dans ses rayons tous les disques de Radio-Canada International $(\mathrm{RCl})$ qui, à part quelques coproductions, ne sont pas disponibles sur le marché commercial. RadioCanada classifie ses acquisitions par compositeur et par genre. À partir du fichier "compositeurs canadiens", il est donc possible de recenser les compositeurs québécois et d'établir la liste de leurs œuvres sur disque, fiche par fiche. Étape suivante: à partir du fichier des commandes de disques cette fois, il est possible d'établir la date de parution approximative des disques qui n'apparaît pas toujours sur le disque lui-même. Un long exercice, donc, mais qui a permis de composer la liste la plus complète possible, nous semble-t-il, des enregistrements sur disque d'œuvres de nos compositeurs, si l'on considère que la discothèque de Radio-Canada a toujours su respecter son mandat face à nos créateurs. Peut-être le catalogue ainsi établi comporte-t-il quelques omissions, mais il serait surprenant qu'elles soient légion!
(1) Sont considérés comme québécois les compositeurs nés au Québec ou y œuvrant depuis au moins dix ans.

(2) Pierre Gamache, directeur du catalogage de la musique à la Bibliothèque nationale du Canada, précise que l'Institut canadien de l'information scientifique et technique du Conseil de recherche du Canada est en train de perfectionner l'accès aux données accumulées aux Archives et que l'on devrait bientôt pouvoir aisément obtenir ce genre d'informations. 
De 1980 à aujourd'hui, ont paru cent dix-huit disques ${ }^{(3)}$ mettant en vedette au moins une œuvre de compositeurs québécois contemporains. En tout, une trentaine d'étiquettes différentes. La production se répartit comme suit:

\begin{tabular}{llr} 
ÉTIQUETTE & ABRÉVIATION & NOMBRE \\
\hline Radio-Canada & RCI & 32 \\
Société Nouvelle d'Enregistrement & SM(CD) & 3 \\
Centredisques & SNE & 21 \\
McGill University Records & CMC & 16 \\
Melbourne & MCGill & 9 \\
Bell Canada & SMLP & 6 \\
empreintes DIGITALes & Bell & 4 \\
Chant du monde & IMED & 3 \\
Kébecdisc & LCD & 2 \\
Divers & KD & 2 \\
TOTAL & & 20
\end{tabular}

Il convient de compléter ces données par les observations suivantes:

1) Ces cent dix-huit disques comprennent chacun un ou plusieurs compositeurs québécois.

2) En tout, soixante-quatre compositeurs, de Serge Arcuri à Claude Vivier, ont au moins une de leurs œuvres gravée sur disque.

3) Trente-deux compositeurs ont une partie de leurs œuvres enregistrées sur $\mathrm{RCl}$.

4) Onze d'entre eux disparaissent complètement du catalogue si l'on exclut les productions non commercialisées de $\mathrm{RCl}$; parmi eux Boudreau, Brégent, Frenette, Gonneville et Lalonde.

5) Quatre œuvres ont été enregistrées deux fois, par des compagnies différentes:

Songs of contemplation d'Alexander Brott,

Mouvement symphonique $n^{\circ} 2$ de Roger Matton,

Divertissement de Pierre Mercure,

Scherzo d'André Prévost.

6) On peut identifier les «compositeurs-vedettes» de la décennie. Nous établissons ce palmarès de deux manières : en tenant compte ou non de $\mathrm{RCl}$, afin de souligner le rôle joué par cette étiquette au moment où elle vient d'être supprimée, ce dont nous reparlerons plus loin.
(3) Ce nombre ne tient pas compte du projet d'Anthologie de la musique canadienne dans lequel plusieurs des disques dénombrés sont réédités. 
a) en incluant $\mathrm{RCl}$ :

$\begin{array}{ll}\text { C. Vivier } & 11 \\ \text { J. Hétu } & 10\end{array}$

$\begin{array}{ll}\text { A. Prévost } & 9 \\ \text { J. Papineau-Couture } & 9 \\ \text { G. Tremblay } & 6 \\ \text { B. Hambreus } & 6 \\ \text { B. Cherney } & 6 \\ \text { M. Longtin } & 6 \\ \text { F. Morel } & 5 \\ \text { J. Rea } & 5\end{array}$

b) en excluant $\mathrm{RCl}$ :

NOM NOMBRE ÉCART

\begin{tabular}{llr}
\hline A. Prévost & 9 & 0 \\
C. Vivier & 7 & -4 \\
J. Hétu & 6 & -4 \\
B. Hambreus & 6 & 0 \\
B. Cherney & 5 & -1 \\
P. Mercure & 4 & 0 \\
G. Tremblay & 4 & -2 \\
J. Papineau-Couture & 4 & -5 \\
B. Mather & 4 & 0 \\
M. Longtin & 4 & -2 \\
T. Brady & 4 & 0 \\
J. Rea & 4 & -1 \\
F. Morel & 3 & -2
\end{tabular}

Une surprise, l'absence de Serge Garant au palmarès: ses œuvres sont surtout sorties sur disques dans les années soixante-dix.

À noter l'importance de RCl pour Papineau-Couture, Hétu et Vivier, les discographies entièrement commercialisées de Prévost, Hambreus, Mercure et Mather, et la débrouillardise du jeune Tim Brady qui a réussi à faire enregistrer quatre de ses œuvres sur des étiquettes commerciales. 
c) Parmi ces "vedettes", les compositeurs suivants ont fait l'objet d'une anthologie: C. Vivier, J. Hétu, A. Prévost, J. Papineau-Couture, G. Tremblay, F. Morel, P. Mercure et B. Mather.

7) La production annuelle de disques, de 1980 à 1990, se répartit comme suit:

$\begin{array}{ccccccccccc}1980 & 1981 & 1982 & 1983 & 1984 & 1985 & 1986 & 1987 & 1988 & 1989 & 1990 \\ 10 & 10 & 11 & 12 & 12 & 9 & 14 & 9 & 19 & 7 & 5\end{array}$

8) Étiquettes étrangères les plus importantes: Chant du monde ( $Y$. Daoust, R. Normandeau), Arion (P. Houdy), REM (J. Landry, A. Reboulot), Chandos (A. Prévost) et Scalen (G. Tremblay). II faut souligner que dans les années soixante et soixante-dix, plusieurs compositeurs ont vu leurs œuvres éditées par des compagnies de réputation internationale telles RCA Victor, Philips, CBS, London, Deutsche Gramophon, Capitol. Mais cela semble beaucoup plus difficile dans les années quatre-vingt.

9) On ne peut qu'être frappé, mais non surpris, de la place qu'occupe $\mathrm{RCl}$ dans l'ensemble de cette production, puisque son mandat a toujours été de donner à l'étranger la meilleure image possible de notre culture. Mais il faut également noter que la production sur disques $\mathrm{RCl}$ d'œuvres de nos compositeurs est moins importante dans les années quatre-vingt que dans les années soixante-dix et que les coproductions avec d'autres compagnies sont pratiquement inexistantes. Par contre, on trouve beaucoup plus d'étiquettes commerciales différentes dans les années quatre-vingt que dans les années soixante-dix, même si la plupart d'entre elles n'ont tenté l'aventure qu'une seule fois.

La lecture de ces données donne à penser. Cent dix-huit disques en une décennie pour le bénéfice de soixante-quatre de notre centaine de compositeurs de musique sérieuse... Y a-t-il de quoi être fier? Et autant que le nombre, les conditions dans lesquelles ces disques sont produits méritent réflexion. Cette sélection par le disque des années quatre-vingt, que signifie-t-elle? En cette année 1990 qui marque la disparition du secteur de production de $\mathrm{RCl}$ et qui devient, par le fait même, un point tournant dans l'histoire de la production de disques d'œuvres québécoises, la question mérite d'être posée.

Dans les sociétés régies par une économie de marché où la rentabilité se mesure essentiellement en dollars, ne nous leurrons pas: la création sérieuse n'existe déjà qu'au prix d'efforts considérables. Les compagnies de disques font difficilement place aux œuvres d'aujourd'hui, si ce n'est par devoir, pour se donner bonne conscience, ou grâce à une subvention. Et, de toute façon, les disques ainsi produits ont toutes les chances d'être rapidement retirés des catalogues. Dans ce contexte, la parution de chacun des disques relevés plus haut est une aventure qui mérite d'être saluée. 


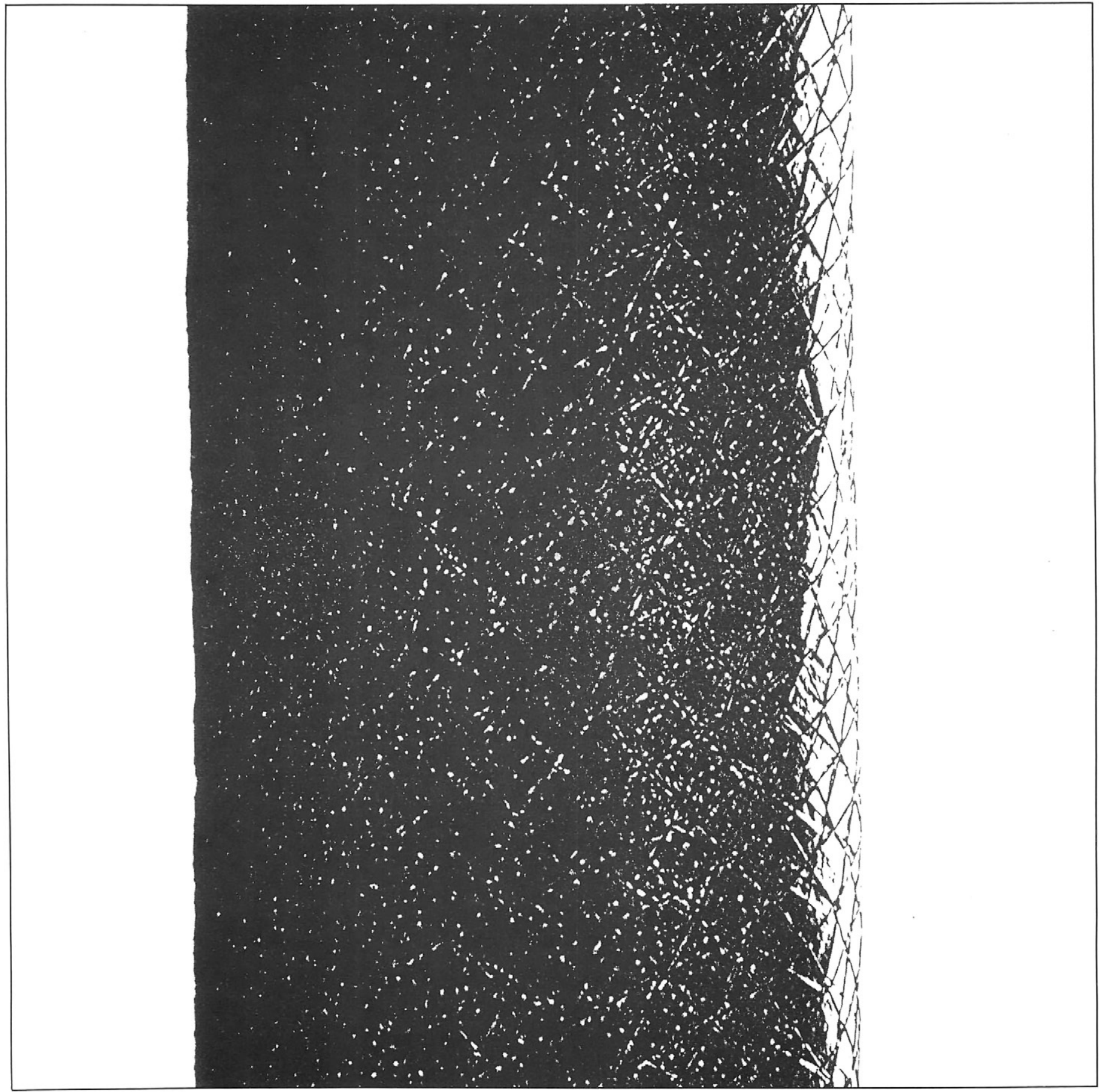


Tout d'abord, voyons brièvement ce qui motive les quatre compagnies les plus productrices de notre catalogue:

\section{McGill University Records (9 disques):}

C'est Paul Pedersen, alors doyen de la Faculté de musique de l'université McGill, à Montréal, qui, en 1977, a fondé cette compagnie qui compte 35 titres à son catalogue. Parmi eux, neuf font place, dans les années quatre-vingt, à nos compositeurs. Grâce aux studios d'enregistrement que possède l'université McGill, les coûts de production sont réduits au minimum. Les fonds manquants sont principalement alloués par le Conseil des arts du Canada et les musiciens contribuent, en se montrant peu exigeants, à la faisabilité du projet. Pour Paul Pedersen, la seule raison d'être des disques McGill repose sur la qualité de la musique et de ses interprètes. Le disque devient une excellente carte de visite pour les intervenants, y compris l'université elle-même. À cet égard, sa rentabilité est incontestable, mais il est inutile de penser augmenter le niveau de production sans un soutien accru des subventions et des ressources de distribution. John Rea, doyen actuel de la Faculté de musique de l'université McGill, ajoute qu'à son avis, une université de ce calibre a une obligation morale envers ses professeurs et, de façon plus globale, envers la musique canadienne en général et québécoise en particulier.

\section{Centredisques ( 16 disques):}

Il est assez normal qu'en 1982, le Centre de musique canadienne se soit lancé dans la production de disques, puisque c'est là la meilleure façon de faire connaître ses membres. Mais, selon Mireille Gagné, directrice du Centre de musique canadienne (CMC) à Montréal, c'est l'une des entreprises les plus ardues du CMC. Pour elle, un disque de haute qualité coûte cher, le résultat ne vaut jamais l'investissement au plan financier, et l'énergie déployée pour un tel projet est démesurée. Au CMC, la chose se complique encore du fait que les projets présentés, soit par un compositeur, soit par les interprètes, ou encore par les radios ou les directeurs des différents centres, sont d'abord soumis à un jury du CMC qui en retient quelques-uns, lesquels sont à leur tour soumis au jury du Conseil des arts du Canada. Une fois les projets sélectionnés, Radio-Canada ou CBC offrent 
le soutien technique nécessaire, mais il reste à trouver des fonds pour la pochette et les autres frais connexes. Certaines fondations font leur part (Laidlaw, Woodland) mais cela devient de plus en plus difficile. Comment peut-on convaincre une compagnie privée de subventionner un produit dont on risque de vendre mille exemplaires, sinon moins, en dix ans?

\section{La Société Nouvelle d'Enregistrement (21 disques):}

On peut comprendre que le Centre de musique canadienne se sente certaines obligations envers ses membres ou qu'une université veville mettre ses professeurs en valeur. Mais qu'un individu, seul, travaille pour la gloire de notre culture, voilà qui mérite, sans aucun doute, un coup de chapeau particulier. Ancien réalisateur au Service des émissions musicales de Radio-Canada, Gilles Poirier a décidé de consacrer sa retraite, non à la pêche ou aux voyages en Floride, mais à la production de disques. SNE voit le jour en 1978 et, déjà, deux compositeurs québécois figurent sur le premier disque. Depuis douze ans, Gilles Poirier connaît à fond tous les rouages de la production d'œuvres québécoises sur disques puisqu'il leur a toujours fait une large place. Conseil des arts du Canada, ministère des Affaires culturelles du Québec, fondations, financement privé, investissement personnel (il vit de sa retraite et ne s'accorde évidemment aucun salaire), il connaît toutes les sources de financement. Les compositeurs ou les interprètes lui soumettent des projets, il en retient une série et, à partir de là, prend en charge toutes les démarches. II soumet de dix à douze demandes à chaque jury du Conseil des arts du Canada qui, pour chaque projet mené à bien, lui fournit en moyenne soixante-cinq pour cent des fonds nécessaires. Ce soutien est relativement faible si l'on considère que les ventes moyennes des disques SNE se situent autour de cinq cents exemplaires par disque. Mais Gilles Poirier résiste, de la façon la plus artisanale, donc la plus économique qui soit, et nos statistiques ci-dessus démontrent que, grâce à sa ténacité et à la petite équipe dévouée qui l'entoure, il est le producteur indépendant qui sert actuellement le mieux nos compositeurs.

\section{Société et Entreprises Radio-Canada (35 disques):}

Les chiffres sont éloquents. La série $S M(C D)$, lancée sur la marché commercial, ne peut se vanter que de trois contributions à l'ensemble de la 
production discographique d'œuvres québécoises depuis 1980. Par contre, le secteur $\mathrm{RCl}$, qui, rappelons-le, avait pour mandat la diffusion de ses disques uniquement auprès des radios étrangères, des ambassades, des universités et de diverses institutions culturelles, à l'exclusion de toute forme de vente commerciale, en a produit à lui seul vingt-six, neuf pour cent de ses disques. Mais aujourd'hui, $\mathrm{RCl}$ n'existe plus comme tel. Son démantèlement, qui en a fait hurler plusieurs au sein de notre milieu musical, était prévisible depuis déjà quelque temps. Monique Grenier, réalisatrice à $\mathrm{RCl}$ pendant de nombreuses années, n'oublie pas que ce secteur, à l'origine un service de Radio-Canada mais relevant de la Division de l'information du ministère des Affaires extérieures du Canada, a été "offert» à Radio-Canada par le gouvernement. Un beau cadeau empoisonné, évidemment sans budget. Radio-Canada n'a donc eu d'autre choix que d'accueillir ce bébé non désiré, mais de là à en assumer la totale responsabilité avec conviction, il y avait un grand pas, aisément infranchissable! Depuis 1946, $\mathrm{RCl}$ a fait de son mieux, dans des conditions jamais idéales. Bien sûr, on peut critiquer le choix des œuvres, la qualité incertaine de certains enregistrements et, par-dessus tout, l'absence de distribution des disques. Mais, précise Monique Grenier, Radio-Canada ne s'est jamais intéressée à cette question, au grand étonnement de tous. Même le fabuleux projet d'Anthologie de la musique canadienne est né non d'une philosophie ou d'une politique culturelle mais, croyez-le ou non, d'un manque d'espace! E†, avec les années, $\mathrm{RCl}$ a dû faire des choix de plus en plus difficiles parmi des compositeurs de plus en plus nombreux avec des budgets de plus en plus serrés. Pas surprenant, donc, qu'on en soit parvenu à la situation présente.

Les années quatre-vingt, c'est donc une dizaine de disques environ par année, dans des conditions de plus en plus difficiles. La quadrature du cercle! D'une part, le disque se doit bien d'exister, puisqu'il constitue une carte de visite exceptionnelle pour nos compositeurs sur la scène nationale et internationale, que ce soit auprès des musiciens, des radios, des festivals ou autres organisations: c'est par lui que l'on peut se construire une histoire musicale. D'autre part, il ne peut exister en termes de marché puisqu'il est évident que, sauf exception, il ne deviendra jamais populaire ${ }^{(4)}$ au sens large du terme, donc économiquement rentable. Sa seule chance de survie véritable est donc, que ce soit au Québec, au Canada ou ailleurs, la mise en place d'une politique générale de production des disques de musique actuelle. On en est loin. L'heure n'est guère à l'optimisme, si l'on en juge par les commentaires des musiciens dont on a retenu les plus éloquents. Pour Denis Gougeon, la situation reflète un flagrant manque de foi dans l'art contemporain. Selon lui, les principaux bailleurs de fonds, que ce soit au niveau fédéral ou provincial, sont les premières victimes de ce vide politique. Walter Boudreau, lui, est amer. II constate la bonne volonté du ministère des Affaires culturelles du Québec qui n'a pas d'argent! Mais il déplore cependant le chauvinisme des organismes subven-
(4) Sur les cent dix-huit disques dénombrés, on compte sur les doigts d'une main ceux qui se sont "bien" vendus. 
tionneurs qui contribue à la mise en place de programmes boiteux : d'un côté, les gouvernements demandent aux producteurs de disques d'être agressifs, de tenter des percées plus vastes sur le marché, y compris le marché international; de l'autre, les subventions sont calculées au prorata du contenu canadien, alors que la seule façon de vendre serait d'utiliser comme locomotives certaines œuvres contemporaines déjà renommées. De plus, si les interprètes ne sont pas canadiens, les portes se ferment presque automatiquement. André Prévost abonde dans le même sens et regrette de voir exclues des programmes de subventions les compagnies internationales d'envergure qui, en plus d'ouvrir aux compositeurs les portes d'un vaste marché, pourraient contribuer à créer, ici, une réelle tradition dans le domaine de la réalisation de disques. Au Conseil des arts du Canada, qui est l'organisme subventionneur le plus généreux en la matière, on est bien conscient des limites du programme d'aide à l'enregistrement, mais il faut bien en fixer, et on accepte mal d'être critiqué alors qu'on cherche à servir avant tout la création d'œuvres canadiennes par des artistes et des compagnies de disques du pays. Avec ces limites, la situation se dégrade constamment, les budgets du programme étant gelés depuis plusieurs années. La production des années quatre-vingt en témoigne puisque, depuis 1988, les parutions sont de plus en plus rares.

Absence de vision, d'où budgets gelés. Restait à concrétiser plus avant l'attitude de nos dirigeants face à la diffusion de la musique actuelle: le démantèlement de $\mathrm{RCl}$ s'en est chargé. On ne peut que se rallier à Laurent Major, réalisateur à Radio-Canada( ${ }^{(5)}$, qui voit dans ce geste la suppression d'un outil essentiel de rayonnement que notre société s'était donné. Certes, les disques ne circulaient pas sur le marché commercial, mais leur impact était loin d'être négligeable. Restait à franchir justement l'étape de commercialisation et elle a été rejetée. C'est un choix de société, une société qui risque de faire bien piètre figure sur la scène internationale si elle ne se donne pas les moyens de se reprendre en main. Il est vrai que $\mathrm{RCl}$ ne disparaît pas entièrement puisque le service de distribution continue d'exister. On achètera les disques publiés par d'autres, et on pourra à nouveau envisager certaines coproductions. Mais, pour Monique Grenier et Gilles Potvin, ce sont là des miettes, puisque le choix d'œuvres sera considérablement réduit. Leur seul espoir est que le réseau français de RadioCanada prenne la relève pour les compositeurs québécois, mais cela ne va pas sans difficultés. Denis Regnaud, chef du Service des émissions musicales, est bien conscient qu'il ne pourra absorber le mandat de $\mathrm{RCl}$. S'il doit enregistrer des disques en studio, il devra alors capter ou produire moins de concerts, et le mandat de la radio ne sera plus respecté. Reste la possibilité des coproductions, à la condition de ne pas oublier que RadioCanada n'assumera pas la plus grande partie des coûts, et qu'on ne pourra compter sur le soutien du gouvernement qui ne croit pas à ce genre d'entreprise non rentable à court terme ${ }^{(6)}$.
(5) Laurent Major a signé plusieurs enregistrements d'œuvres contemporaines sur disques.

(6) Au moment de mettre sous presse, Daniel Vachon, coordonnateur des enregistrements phonogrammes du Service des émissions musicales de Radio-Canada, annonce cependant la parution prochaine des disques suivants :

- réédition en compact de l'Anthologie Serge Garant, chez Doberman:

- œuvres de José Evangelista (Piano concertant, Monidías españolas, Clos de vie, Ô Bali, M. Plume un homme paisible) chez Salabert;

- le deuxième disque du NEM (œuvres de Rozankovic, Mather, Rea et Longtin) sur étiquette UMMUS; 
Les préoccupations de nos dirigeants sont hélas depuis fort longtemps ailleurs: ils misent sur une culture qui s'autofinancerait, selon le modèle américain, mais avec les fondations en moins. D'ailleurs, de quelle culture parle-t-on? De divertissement ou d'art? John Rea ne mâche pas ses mots : $d^{\prime}$ ici un siècle, on saura faire la part des choses entre la bêtise des gouvernements et l'esprit d'entreprise de certains artistes.

Car, et c'est peut-être la seule lueur d'espoir, certains artistes résistent contre vents et marées. Denis Gougeon est persuadé que la seule façon pour les compositeurs de s'en sortir est de s'infiltrer à tous les niveaux où I'on prend les décisions. Inutile de rêver à la prise en charge qui permettrait au génie créateur de s'exprimer en toute quiétude. C'est un peu la philosophie qui sous-tend la naissance, en 1990, de la nouvelle étiquette empreintes DIGITALes: une compagnie qui mise sur l'esprit d'entreprise de ses fondateurs-compositeurs-producteurs-hommes d'affaires, sur des coûts de production très bas (facilités dans le cas de la musique électroacoustique) et sur un produit de très haute qualité. Claude Schryer et Jean-François Denis voient dans cette initiative l'extension de leur travail de création et, dans ce sens-là, c'est un investissement rentable. Selon eux, la clé du succès réside dans l'établissement d'un solide réseau international de distribution et ils y consacrent tous leurs efforts. Lorraine Vaillancourt renchérit: le disque est impératif, non seulement pour les compositeurs mais pour les interprètes et pour le public. Et rien ne sert de se plaindre, il faut savoir se prendre en main. Plus un marché est petit, plus il faut se battre, et à force de démarches, on finit par se faire écouter. L'absence de politique culturelle réelle est tragique, mais c'est à tous de s'unir pour que cela change.

Exceptionnellement, on verra toujours quelques compagnies prendre des risques, par devoir ou pour suivre certaines modes, et on parviendra parfois à convaincre certaines entreprises privées de faire leur part (c'est le cas de Bell Canada avec les disques des tournées de l'Orchestre du conservatoire de musique du Québec qui inscrit toujours une œuvre québécoise à son programme). On pourra toujours compter sur les universités pour faire la promotion sur disque de leurs musiciens. (Saluons la naissance de l'étiquette UMMUS à la Faculté de musique de l'Université de Montréal.) Bien sûr, on déploiera des trésors d'imagination pour faire «passer» sans trop de mal ces œuvres contemporaines. Mais est-ce suffisant?

Avec la disparition des productions de $\mathrm{RCl}$ qui, de par son mandat, donnait au moins une chance à bon nombre de compositeurs d'exister sur disque (n'oublions pas que, pour les années quatre-vingt, onze compositeurs disparaissent du catalogue avec la fermeture du RCl), on a presque envie de crier: "Que le plus débrouillard gagne!» Mais ne soyons pas naîfs. Est-ce ainsi que l'on bâtit une culture, à partir d'initiatives isolées, d'investissements financiers personnels et de bénévolat? Peut-on vraiment se passer de structures sous-jacentes fortes et organisées? La production
- le premier enregistrement mondial de l'Opéra Kopernikus de Claude Vivier, dirigé par Lorraine Vaillancourt;

- un disque de la SMCQ (œuvres de Boudreau et Rea);

- un disque SRC de l'Ensemble vocal Tudor (Hétu, Vivier, Prévost, Patriquin);

- un disque SRC de l'Orchestre Métropolitain (œuvres de Boudreau et Vivier, et les créations du concert inaugural de Montréal Musiques Actuelles - Bouchard, Cherney, Gougeon), dirigé par Walter Boudreau.

Radio-Canada entend donc bien explorer toutes les possibilités de travailler pour nos compositeurs en fonction, bien sûr, de ses ressources financières. 
de disques des années quatre-vingt-dix risque bien, dans les conditions actuelles, d'être encore plus réduite que celle des années quatre-vingt, à moins que les autorités ne se décident enfin à établir une véritable politique de soutien aux efforts considérables de nos compositeurs, de nos interprètes, de nos gens de radio et de nos producteurs. Mais se doter d'une telle politique, c'est assumer la responsabilité de la création dans une société. Et c'est un défi dont l'enjeu est élevé, puisque c'est la force d'une culture qui donne pouvoir et liberté. Qui saura le relever ${ }^{(7)}$ ?
(7) Cet article a été rédigé en juillet 1990 et mis à jour en janvier 1991. Nous adressons nos remerciements chaleureux au personnel de la discothèque de Radio-Canada à Montréal, en particulier à Lucie Tremblay et à Denis Girard, à l'équipe de l'émission "Musique actuelle» de Radio-Canada FM, et aux personnes qui ont accepté de nous rencontrer et dont nous avons rapporté les propos. 


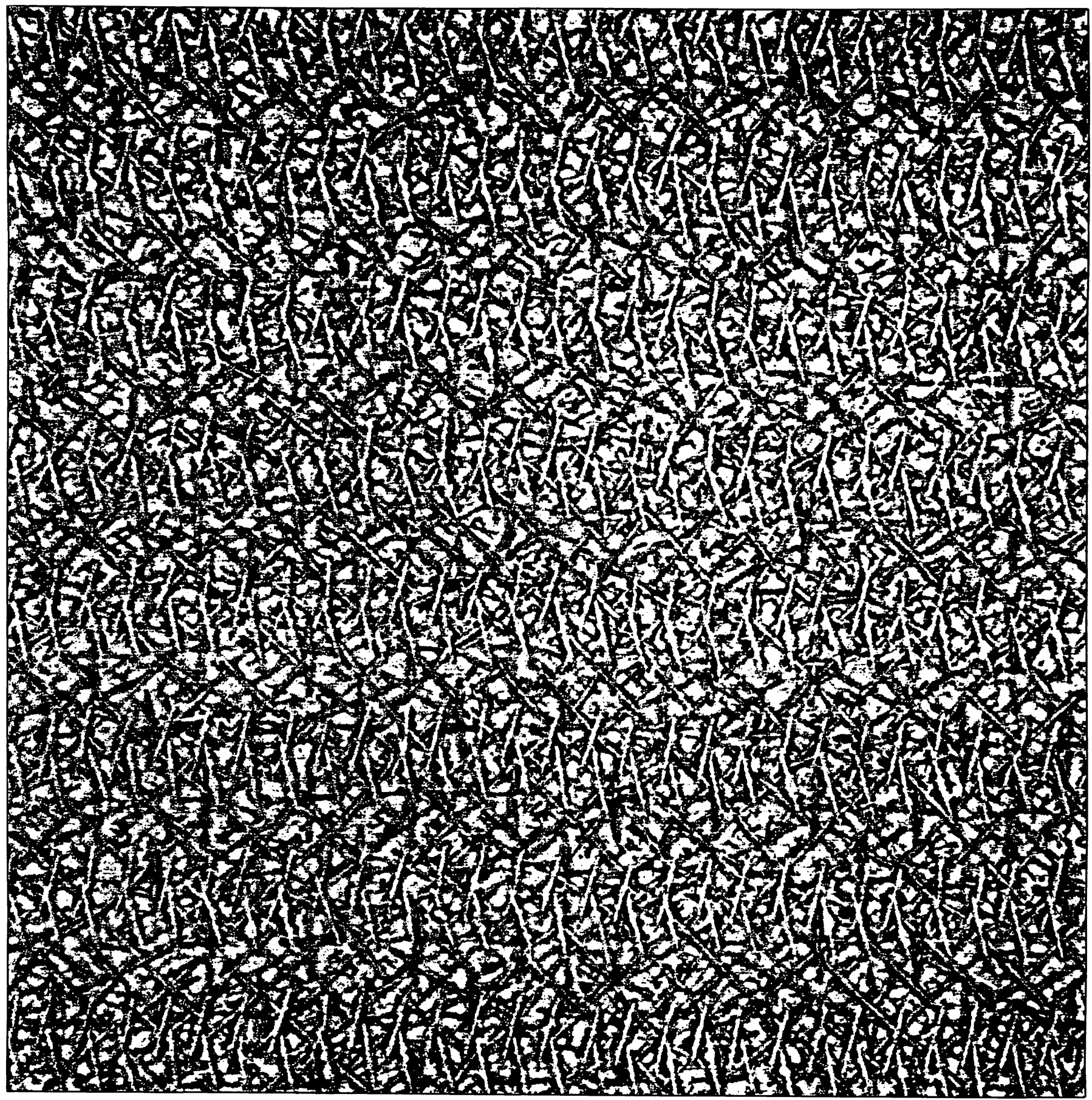

